\title{
Conical maximal regularity for elliptic operators via Hardy spaces
}

\author{
YI HUANG
}

\begin{abstract}
We give a technically simple approach to the maximal regularity problem in parabolic tent spaces for second order, divergence form, complex valued elliptic operators. By using the associated Hardy space theory combined with certain $L^{2}-L^{2}$ off-diagonal estimates, we reduce the tent space boundedness in the upper half-space to the reverse Riesz inequalities in the boundary space. This way, we also improve recent results obtained by P. Auscher et al.
\end{abstract}

Mathematics Subject Classification 2010: 42B37; 47D06; 42B35; 42B20. Keywords: Maximal regularity operators; tent spaces; elliptic operators; Hardy spaces; off-diagonal decay; maximal $L^{p}$-regularity.

\begin{tabular}{lll}
\hline & Introduction & 1
\end{tabular}

\begin{tabular}{|lll}
2 & Elliptic operators and Hardy spaces & 4
\end{tabular}

\begin{tabular}{|l|l|l|l|l}
\hline 3 & Proof of Theorem 1.1 & 5
\end{tabular}

\begin{tabular}{ll}
\hline Acknowledgements & 10
\end{tabular}

\begin{tabular}{ll}
\hline References & 10
\end{tabular}

\section{Introduction}

Let $\mathbb{R}_{+}^{1+n}$ be the upper half-space $\mathbb{R}_{+} \times \mathbb{R}^{n}$ with $\mathbb{R}_{+}=(0, \infty)$ and $n \in \mathbb{N}_{+}=$ $\{1,2, \cdots\}$. Define the tent space $T_{\mathrm{par}}^{p}, \frac{n}{n+1}<p<\infty$, as the space of all 
locally square integrable functions on $\mathbb{R}_{+}^{1+n}$ such that

$$
\|F\|_{T_{\mathrm{Par}}^{p}}:=\left(\int_{\mathbb{R}^{n}}\left(\iint_{\mathbb{R}_{+}^{1+n}} \frac{\mathbf{1}_{B\left(x, t^{\frac{1}{2}}\right)}(y)}{t^{\frac{n}{2}}}|F(t, y)|^{2} d t d y\right)^{\frac{p}{2}} d x\right)^{\frac{1}{p}}<\infty .
$$

The scale $T_{\mathrm{par}}^{p}, \frac{n}{n+1}<p<\infty$, is a parabolic analogue of the tent spaces introduced by R. R. Coifman, Y. Meyer and E. M. Stein in CMS85].

Let $A=A(x)$ be an $n \times n$ matrix of complex $L^{\infty}$ coefficients, defined on $\mathbb{R}^{n}$, and satisfying the ellipticity (or "accretivity") condition

$$
\lambda|\xi|^{2} \leq \operatorname{Re} A \xi \cdot \bar{\xi} \text { and }|A \xi \cdot \bar{\zeta}| \leq \Lambda|\xi||\zeta|
$$

for $\xi, \zeta \in \mathbb{C}^{n}$ and for some $\lambda, \Lambda$ such that $0<\lambda \leq \Lambda<\infty$. Let

$$
L:=-\operatorname{div} A \nabla
$$

(its precise definition will be recalled in next section). Consider the associated forward maximal regularity operator $\mathbf{M}_{L}^{+}$given by

$$
\mathbf{M}_{L}^{+}(F)_{t}:=\int_{0}^{t} L e^{-(t-s) L} F_{s} d s
$$

originally defined on $F \in L^{2}\left(\mathbb{R}_{+} ; \mathbf{D}(L)\right)$. Here $\mathbf{D}(L)$ is the domain of $L$ in $L^{2}\left(\mathbb{R}^{n}\right)$ and $F_{s}=F(s, \cdot)$. By a classical result of L. de Simon dS64, $\mathbf{M}_{L}^{+}$ extends to a bounded operator on $L^{2}\left(\mathbb{R}_{+} ; L^{2}\left(\mathbb{R}^{n}\right)\right)$. By Fubini's theorem

$$
T_{\text {par }}^{2}\left(\mathbb{R}_{+}^{1+n}\right) \simeq L^{2}\left(\mathbb{R}^{n} ; L^{2}\left(\mathbb{R}_{+}\right)\right) \text {. }
$$

For $p$ different from 2, the analogous equivalence of (4) between $T_{\mathrm{par}}^{p}\left(\mathbb{R}_{+}^{1+n}\right)$ and $L^{p}\left(\mathbb{R}^{n} ; L^{2}\left(\mathbb{R}_{+}\right)\right)$breaks down. We shall refer to the maximal regularity (namely, the boundedness of $\mathbf{M}_{L}^{+}$) in $T_{\text {par as conical maximal regularity for }}^{p}$ the reason that (parabolic) cones are involved in defining tent spaces in (1).

The maximal regularity operator $\mathbf{M}_{L}^{+}$is a typical example of singular integral operators with operator valued kernels. Let $1 \leq p \leq 2$. Let

$$
\operatorname{dist}\left(E, E^{\prime}\right):=\inf \left\{|x-y|: x \in E, y \in E^{\prime}\right\} .
$$


We shall say that a class of uniformly $L^{2}=L^{2}\left(\mathbb{R}^{n}\right)$ bounded kernels $\{T(t)\}_{t>0}$ satisfies the $L^{p}-L^{2}$ off-diagonal decay with some order $M \in \mathbb{N}_{+}$, if we have

$$
\left\|\mathbf{1}_{E^{\prime}} T(t) \mathbf{1}_{E} f\right\|_{L^{2}} \lesssim t^{-\frac{n}{2}\left(\frac{1}{p}-\frac{1}{2}\right)}\left(1+\frac{\operatorname{dist}\left(E, E^{\prime}\right)^{2}}{t}\right)^{-M}\left\|\mathbf{1}_{E} f\right\|_{L^{p}}
$$

for all Borel sets $E, E^{\prime} \subset \mathbb{R}^{n}$, all $t>0$ and all $f \in L^{p} \cap L^{2}$. We shall say $\{T(t)\}_{t>0}$ satisfies the $L^{p}-L^{2}$ off-diagonal decay if it satisfies the $L^{p}-$ $L^{2}$ off-diagonal decay with any order $M \in \mathbb{N}_{+}$. Denote by $p_{-}=p_{-}(L)$ the infimum of $p$ for which the heat semigroup $\left\{e^{-t L}\right\}_{t>0}$ satisfies the $L^{p}-L^{2}$ off-diagonal decay. Define the index

$$
\left(p_{-}\right)_{*}:=\frac{n p_{-}}{n+p_{-}}
$$

For $L=-\Delta=-\operatorname{div} \nabla$, one has $p_{-}=1$ and $1_{*}=\frac{n}{n+1}$.

Our main result in this letter reads as follow.

Theorem 1.1. Let $L=-\operatorname{div} A \nabla$ with $A$ satisfying (2) and $p_{-}$defined as in (6). Then, for $p \in\left(\left(p_{-}\right)_{*}, 2\right]$, the maximal regularity operator $\mathbf{M}_{L}^{+}$defined as in (3) extends to a bounded operator on $T_{\text {par. }}^{p}$.

We end the introduction with several remarks.

Remark 1.2. Under the assumption $\left(p_{-}\right)_{*}<1$ Theorem 1.1 is first proved in [AKMP12, Theorem 3.1] (with $m=2, \beta=0$ and $q$ close to $p_{-}$in their statement). Indeed, we note that $\left(p_{-}\right)_{*}<1$ is equivalent to $\left(p_{-}\right)^{\prime}>n$, where $\left(p_{-}\right)^{\prime}$ is the dual exponent of $p_{-}$. A threshold condition essentially the same as $\left(p_{-}\right)^{\prime}>n$ is used in [AKMP12.

A general framework of singular integral operators on tent spaces is also presented in AKMP12. Their method is heavily based on the $L^{p}-L^{2}$ offdiagonal decay of the family $\left\{t L e^{-t L}\right\}_{t>0}$ for $p \in\left(p_{-}, 2\right)$. Note that they already improved the previous result in AMP12, the $T_{\text {par-boundedness of }}^{p}$ $\mathbf{M}_{L}^{+}$for $p \in\left(2_{*}, 2\right]$, which assumes $L^{2}-L^{2}$ off-diagonal decay only.

Here we shall give a technically simple approach to Theorem 1.1 by using the well-established $L$-associated Hardy space theory combined (mainly) with $L^{2}-L^{2}$ off-diagonal decay of $\left\{t L e^{-t L}\right\}_{t>0}$. 
Remark 1.3. The motivation of the following reduction scheme

(Operator theory on tent spaces) $\longrightarrow$ (Hardy space theory),

which is involved in our proof of Theorem 1.1, comes from the study of conical maximal regularity (in elliptic tent spaces) for first order perturbed Dirac operators. See [Hua15, Chap. 5]. Furthermore, the motivation of considering such conical (elliptic) maximal regularity estimates is suggested by their applications to boundary value elliptic problems (see [AA11 for example). In parabolic case, the conical maximal regularity results have already proven to be useful in various settings (see for example AvNP14, AF15]).

Remark 1.4. Though the singularity of the integral operator $\mathbf{M}_{L}^{+}$is at $s=t$, the most involved part turns out to be the estimation of tent space norms when $s \rightarrow 0$. For more explanations concerning the "singularity" pertaining to singular integral operators and maximal regularity operators on tent spaces, see [AKMP12, Remark 3.6] and [AF15, Remark 5.23].

Remark 1.5. Theorem 1.1 also extends to higher order elliptic operators. Then one changes correspondingly the homogeneity of tent spaces and offdiagonal decay in (5). We leave this issue to the interested reader.

\section{Elliptic operators and Hardy spaces}

We give some preliminary materials needed in the proof of Theorem 1.1 .

Let $A$ satisfy (2). We define the divergence form elliptic operator

$$
L f:=-\operatorname{div}(A \nabla f)
$$

which we interpret in the sense of maximal-accretive operators via a sesquilinear form. That is, $\mathbf{D}(L)$ is the largest subspace contained in $W^{1,2}$ for which

$$
\left|\int_{\mathbb{R}^{n}} A \nabla f \cdot \nabla g\right| \leq C\|g\|_{2}
$$

for all $g \in W^{1,2}$ and we set $L f$ by

$$
\langle L f, g\rangle=\int_{\mathbb{R}^{n}} A \nabla f \cdot \overline{\nabla g}
$$


for $f \in \mathbf{D}(L)$ and $g \in W^{1,2}$. Thus defined, $L$ is a maximal-accretive operator on $L^{2}$ and $\mathbf{D}(L)$ is dense in $W^{1,2}$. Furthermore, $L$ has a square root, denoted by $L^{1 / 2}$ and defined as the unique maximal-accretive operator such that

$$
L^{1 / 2} L^{1 / 2}=L
$$

as unbounded operators (see [Kat76, p. 281]).

For $L$ as formulated above, the development of an $L$-associated Hardy space theory, was taken in HM09 (and independently in AMR08 in a different geometric setting), in which the authors considered the model case $H_{L}^{1}\left(\mathbb{R}^{n}\right)$. In presence of pointwise heat kernel bounds, see [DY05. The definition of $H_{L}^{1}$ given in [HM09, AMR08 can be extended immediately to $\frac{n}{n+1}<p \leq 2$ (see [HMM11]). To this end, consider the (conical) square function associated with the heat semigroup generated by $L$

$$
\mathbf{S}_{L}(f)(x):=\left(\iint_{\Gamma(x)}\left|t^{2} L e^{-t^{2} L} f(y)\right|^{2} \frac{d t d y}{t^{1+n}}\right)^{\frac{1}{2}}, \quad x \in \mathbb{R}^{n},
$$

where, as usual,

$$
\Gamma(x)=\left\{(t, y) \in \mathbb{R}_{+}^{1+n}:|x-y|<t\right\}
$$

is a non-tangential cone with vertex at $x \in \mathbb{R}^{n}$. As in [HM09, HMM11], we define $H_{L}^{p}\left(\mathbb{R}^{n}\right)$ for $\frac{n}{n+1}<p \leq 2$ as the completion of

$$
\left\{f \in L^{2}\left(\mathbb{R}^{n}\right): \mathbf{S}_{L}(f) \in L^{p}\left(\mathbb{R}^{n}\right)\right\}
$$

in the quasi-norm

$$
\|f\|_{H_{L}^{p}\left(\mathbb{R}^{n}\right)}:=\left\|\mathbf{S}_{L}(f)\right\|_{L^{p}\left(\mathbb{R}^{n}\right)} .
$$

We will not get into the dual side $(p>2)$ of the Hardy space theory.

For $L^{2}-L^{2}$ off-diagonal decay related to $\left\{e^{-s L}, s L e^{-s L}, \sqrt{s} \nabla e^{-s L}\right\}_{s>0}$, and other holomorphic functions of $L$ (for example $\left(I-e^{-s L}\right)^{\sigma}$ with $\sigma>0$ ), we refer to Chapter 2 of the memoir [Aus07.

\section{Proof of Theorem 1.1}

Note that the extension of $\mathbf{M}_{L}^{+}$will divided into two steps: first from $F \in$ $L^{2}\left(\mathbb{R}_{+} ; \mathbf{D}(L)\right)$ to $T_{\text {par }}^{2}$, and then for $\frac{n}{n+1}<p<2$, from $T_{\text {par }}^{2} \cap T_{\text {par }}^{p}$ to $T_{\text {par }}^{p}$. 
First we split the operator $\mathbf{M}_{L}^{+}$: for $\ell \in \mathbb{N}_{+}$large, set

$$
\mathbf{R}_{L}^{\ell}:=\mathbf{M}_{L}^{+}-\mathbf{V}_{L}^{\ell},
$$

where for $F \in L^{2}\left(\mathbb{R}_{+} ; \mathbf{D}(L)\right)$, the singular part $\mathbf{R}_{L}^{\ell}$ is given formally by

$$
\mathbf{R}_{L}^{\ell}(F)_{t}=\int_{0}^{t} L e^{-(t-s) L}\left(I-e^{-2 s L}\right)^{\ell} F_{s} d s,
$$

and the regular part is defined by

$$
\mathbf{V}_{L}^{\ell}=\sum_{k=1}^{\ell}\left(\begin{array}{l}
\ell \\
k
\end{array}\right) \mathbf{V}_{L, k}
$$

with

$$
\mathbf{V}_{L, k}(F)_{t}:=\int_{0}^{t} L e^{-(t+(2 k-1) s) L} F_{s} d s, \quad t \in \mathbb{R}_{+} .
$$

For the above binomial sum $\mathbf{V}_{L}^{\ell}$, it suffices to consider $\mathbf{V}_{L}:=\mathbf{V}_{L, 1}$.

Let $2 \mathbb{N}_{+}=\{2,4, \cdots\}$. We have the following observation.

Lemma 3.1. For $\ell \in 2 \mathbb{N}_{+}$and $\frac{\ell}{2}>\frac{1}{2}+\frac{n}{4}$, the operator $\mathbf{R}_{L}^{\ell}$, as given in (9) through (8), extends to a bounded operator on $T_{\text {par }}^{p}$ for any $\frac{n}{n+1}<p \leq 2$.

Proof. The $T_{\text {par }}^{2}$-boundedness is de Simon's theorem plus the uniform $L^{2}$ boundedness of $\left\{\left(I-e^{-2 s L}\right)^{\ell}\right\}_{s>0}$. By interpolation it suffices to consider $\frac{n}{n+1}<p \leq 1$, and this follows from Lemma 3.4 and Lemma 3.5 of [AKMP12] in the particular case $m=2, \beta=0$ and $q=2$. 1 Indeed, first we can decompose the operator $\mathbf{R}_{L}^{\ell}$ as in [AKMP12] in the way

$$
\begin{aligned}
\mathbf{R}_{L}^{\ell}(F)_{t}= & \int_{t / 2}^{t} L e^{-(t-s) L}\left(I-e^{-2 s L}\right)^{\ell} F_{s} d s \\
& +\int_{0}^{t / 2} L e^{-(t-s) L}\left(I-e^{-2 s L}\right)^{\ell} F_{s} d s=: I+I I .
\end{aligned}
$$

\footnotetext{
${ }^{1}$ We point out that one can also prove this lemma by adapting directly the arguments for Lemma 3.4 of AKMP12] (see [Hua15] for details).
} 
Here we view $\mathcal{T}_{1}=\left\{\left(I-e^{-2 s L}\right)^{\ell}\right\}_{s>0}$ as an operator on $T_{\mathrm{par}}^{p}$ given by

$$
\mathcal{T}_{1}: F \mapsto \mathcal{T}_{1}(F)_{s}:=\left(I-e^{-2 s L}\right)^{\ell} F_{s},
$$

with the similar interpretation for $\mathcal{T}_{2}=\left\{\left(I-e^{-2 s L}\right)^{\ell} /(s L)^{\frac{\ell}{2}}\right\}_{s>0}$ in

$$
\begin{aligned}
& L e^{-(t-s) L}\left(I-e^{-2 s L}\right)^{\ell} \\
& \quad=\left(\frac{s}{t-s}\right)^{\frac{\ell}{2}} L((t-s) L)^{\frac{\ell}{2}} e^{-(t-s) L} \frac{\left(I-e^{-2 s L}\right)^{\ell}}{(s L)^{\frac{\ell}{2}}} .
\end{aligned}
$$

Note that $t-s \sim t$ when $s<t / 2$. Therefore, to obtain the $T_{\mathrm{par}}^{p}$-boundedness of $\mathbf{R}_{L}^{\ell}$ for $\frac{n}{n+1}<p \leq 1$, we can use Lemma 3.4 of AKMP12 together with the $T_{\text {par-boundedness of }}^{p} \mathcal{T}_{1}$ to estimate $I$, and use Lemma 3.5 of [AKMP12]

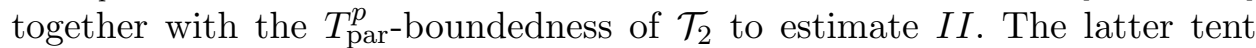
space boundedness results on $\mathcal{T}_{i}, i=1,2$, are implied by their $L^{2}-L^{2}$ offdiagonal decay with order at least $\frac{\ell}{2}$, which satisfies the condition

$$
\frac{\ell}{2}>\frac{1}{2}+\frac{n}{4}=\frac{n}{2}\left(\frac{1}{\frac{n}{n+1}}-\frac{1}{2}\right) .
$$

This implication can be easily verified via the extrapolation method on tent spaces through atomic decompositions. Note that we also need the condition $\frac{\ell}{2}>\frac{1}{2}+\frac{n}{4}$ in $\left(\frac{s}{t-s}\right)^{\frac{\ell}{2}} \sim\left(\frac{s}{t}\right)^{\frac{\ell}{2}}$ when applying Lemma 3.5 of [AKMP12].

Next we rewrite the operator $\mathbf{V}_{L}$ in the following way:

$$
\mathbf{V}_{L}(F)_{t}=-\widetilde{\mathbf{V}}_{L}(F)_{t}+\mathbf{I}_{L}(F)_{t}, \quad t \in \mathbb{R}_{+},
$$

where for $F \in L^{2}\left(\mathbb{R}_{+} ; \mathbf{D}(L)\right)$, the backward part $\widetilde{\mathbf{V}}_{L}$ is defined by

$$
\tilde{\mathbf{V}}_{L}(F)_{t}:=\int_{t}^{\infty} L e^{-(t+s) L} F_{s} d s, \quad t \in \mathbb{R}_{+}
$$

and the trace part $\mathbf{I}_{L}$ is defined by

$$
\mathbf{I}_{L}(F)_{t}:=\int_{0}^{\infty} L e^{-(t+s) L} F_{s} d s=\sqrt{L} e^{-t L} \int_{0}^{\infty} \sqrt{L} e^{-s L} F_{s} d s .
$$

We used the square root property $\sqrt{L} \sqrt{L}=L$ recalled in (7). 
Lemma 3.2. The integral operator $\widetilde{\mathbf{V}}_{L}$ as given in (11) extends to a bounded operator on $T_{\text {par }}^{p}$ for any $\frac{n}{n+1}<p \leq 2$. ${ }^{2}$

Proof. This is a consequence of a more general claim found in [AKMP12, Proposition 3.7], again corresponding to the case $m=2, \beta=0$ and $q=2$. Indeed, [AKMP12, Proposition 3.7] deals with a counterpart to $\mathbf{M}_{L}^{+}$, namely, the backward maximal regularity operator

$$
\mathbf{M}_{L}^{-}(F)_{t}:=\int_{t}^{\infty} L e^{-(s-t) L} F_{s} d s
$$

where $F \in L^{2}\left(\mathbb{R}_{+} ; \mathbf{D}(L)\right)$ and they use the splitting

$$
\begin{aligned}
\mathbf{M}_{L}^{-}(F)_{t}= & \int_{t}^{2 t} L e^{-(s-t) L} F_{s} d s \\
& +\int_{2 t}^{\infty} L e^{-(s-t) L} F_{s} d s=: I I I+I V .
\end{aligned}
$$

We only need to use those arguments in proving AKMP12, Proposition 3.7] with $I V$ involved, since $s-t \sim s$ when $s>2 t$, which is equivalent to that $s+t \sim s$ when $s>t$ in our setting. We omit the details.

Now we use the $L$-associated Hardy spaces, which we recalled in Section 2 , to treat the trace part $\mathbf{I}_{L}$. First, from the conical square function estimates (see [HMM11, Proposition 4.9]), one has, for $\frac{n}{n+1}<p \leq 2$

$$
\left\|\sqrt{L} e^{-t L} \int_{0}^{\infty} \sqrt{L} e^{-s L} F_{s} d s\right\|_{T_{\mathrm{Par}}^{p}} \lesssim\left\|\int_{0}^{\infty} \sqrt{L} e^{-s L} F_{s} d s\right\|_{H_{L}^{p}}
$$

for $F \in L^{2}\left(\mathbb{R}_{+} ; \mathbf{D}(L)\right)$. Next, from the reverse Riesz inequalities (see [HMM11, Proposition 5.17]), one has, for $p \in\left(\left(p_{-}\right)_{*}, 2\right]$

$$
\|\sqrt{L} f\|_{H_{L}^{p}} \lesssim\|\nabla f\|_{H^{p}}
$$

\footnotetext{
${ }^{2}$ As we will see in the proof, the lemma also holds for any $0<p \leq 2$. But that does not help in proving Theorem 1.1 .
} 
for $f \in L^{2}$, hence one further has, for $p \in\left(\left(p_{-}\right)_{*}, 2\right]$

$$
\left\|\int_{0}^{\infty} \sqrt{L} e^{-s L} F_{s} d s\right\|_{H_{L}^{p}} \lesssim\left\|\int_{0}^{\infty} \nabla e^{-s L} F_{s} d s\right\|_{H^{p}} .
$$

Here, as usual, we make the convention $H^{p}=L^{p}$ for $p>1.3$

For $F \in T_{\text {par }}^{2}$ consider the sweeping operator

$$
\pi_{L}(F):=\int_{0}^{\infty} \nabla e^{-s L} F_{s} d s
$$

An equivalent formulation of the Kato square root estimate for $L^{*}$ (see AHL+02) is the following square function estimate

$$
\iint_{\mathbb{R}_{+}^{1+n}}\left|e^{-t L^{*}} \operatorname{div} \vec{F}(y)\right|^{2} d t d y \lesssim\|\vec{F}\|_{2}^{2}
$$

for all $\vec{F} \in L^{2}\left(\mathbb{R}^{n} ; \mathbb{C}^{n}\right)$, hence the mapping given by

$$
\mathbb{Q}_{L^{*}}: \vec{F} \mapsto \mathbb{Q}_{L^{*}}(\vec{F})(t, y):=\left(e^{-t L^{*}} \operatorname{div} \vec{F}\right)(y)
$$

is bounded from $L^{2}\left(\mathbb{R}^{n} ; \mathbb{C}^{n}\right)$ to $T_{\text {par }}^{2}$. Thereby, we see that $\pi_{L}: T_{\text {par }}^{2} \rightarrow L^{2}$ is a bounded operator by duality with $\mathbb{Q}_{L^{*}}$.

Recall that, a $T_{\mathrm{par}}^{p}$-atom $A$ supported in the parabolic Carleson cylinder

$$
\operatorname{Cyl}(B):=\left(0, r_{B}^{2}\right) \times B
$$

for some ball $B \subset \mathbb{R}^{n}$ (with radius $r_{B}$ ), satisfies the size estimate

$$
\|A\|_{T_{\text {par }}^{2}} \leq|B|^{-\left(\frac{1}{p}-\frac{1}{2}\right)} .
$$

We have the following result on $\pi_{L}$.

\footnotetext{
${ }^{3}$ We remark that in AF15, Lemma 5.21] a variant of $\mathbf{I}_{L}$ is treated in a similar way, with informative connections to the Hardy space theory associated with the first order perturbed Dirac operators as alluded in Remark 1.3
} 


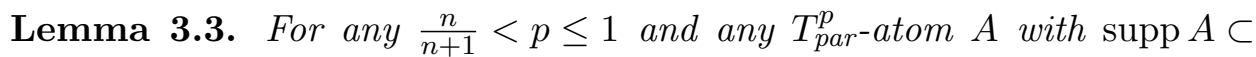
$C y l(B)$ for some ball $B \subset \mathbb{R}^{n}$ (with radius $r_{B}$ ), then we have that

$$
m:=\pi_{L}(A)=\int_{0}^{r_{B}^{2}} \nabla e^{-s L} A_{s} d s
$$

satisfies the uniform estimate

$$
\|m\|_{H^{p}} \lesssim 1
$$

Hence $\pi_{L}$ extends to a bounded operator from $T_{p a r}^{p}$ to $H^{p}$ for $\frac{n}{n+1}<p \leq 2$.

Proof. For $m=\pi_{L}(A)$ with $A$ being $T_{\text {par-atoms, }}^{p} \frac{n}{n+1}<p \leq 1$, and by adapting [CMS83, Théorème 3] and [CMS85, Theorem 6], (13) follows from the $L^{2}-L^{2}$ off-diagonal decay for the heat semigroup $\left\{e^{-s L}\right\}_{s>0}$ and the gradient family $\left\{\sqrt{s} \nabla e^{-s L}\right\}_{s>0}$, the size estimate 12 and the Coifman-Weiss molecular theory for $H^{p}$. Then for $\frac{n}{n+1}<p \leq 1, \pi_{L}$ extends to a bounded operator from $T_{\mathrm{par}}^{p}$ to $H^{p}$, and by interpolation, $\pi_{L}$ extends to a bounded operator from $T_{\text {par }}^{p}$ to $H^{p}$ for $\frac{n}{n+1}<p \leq 2$.

With the splittings $(8)$ and $(10)$, together with the conditions $\ell \in 2 \mathbb{N}_{+}$ and $\frac{\ell}{2}>\frac{1}{2}+\frac{n}{4}$, and using Lemmata $3.1,3.2$ and 3.3 in order, the proof of Theorem 1.1 (with $p \in\left(\left(p_{-}\right)_{*}, 2\right]$ ) is then concluded.

\section{Acknowledgements}

This research is supported in part by the ANR project "Harmonic Analysis at its Boundaries", ANR-12-BS01-0013-01. I am indebted to my Ph.D advisor Pascal Auscher who motivated me to work on this subject.

\section{References}

[Aus07] Pascal Auscher. On necessary and sufficient conditions for $L^{p}$-estimates of Riesz transforms associated to elliptic operators on $\mathbb{R}^{n}$ and related estimates. Mem. Amer. Math. Soc., 186(871):xviii+75, 2007.

[AA11] Pascal Auscher and Andreas Axelsson. Weighted maximal regularity estimates and solvability of non-smooth elliptic systems I. Invent. Math., 184(1):47-115, 2011. 
"Hua-MaxRegHardy" — 2016/4/15 — 8:49 — page 11 — \#11

Conical maximal regularity \& Hardy spaces

[AF15] Pascal Auscher and Dorothee Frey. On well-posedness of parabolic equations of Navier-Stokes type with $B M O^{-1}\left(\mathbb{R}^{n}\right)$ data. Journal of the Institute of Mathematics of Jussieu, 2015.

[AHL+02] Pascal Auscher, Steve Hofmann, Michael Lacey, Alan McIntosh, and Ph. Tchamitchian. The solution of the Kato square root problem for second order elliptic operators on $\mathbb{R}^{n}$. Ann. of Math. (2), 156(2):633-654, 2002.

[AKMP12] Pascal Auscher, Christoph Kriegler, Sylvie Monniaux, and Pierre Portal, Singular integral operators on tent spaces, J. Evol. Equ. 12 (2012), no. 4, 741-765.

[AMP12] Pascal Auscher, Sylvie Monniaux, and Pierre Portal, The maximal regularity operator on tent spaces, Commun. Pure Appl. Anal. 11 (2012), no. 6, 2213-2219.

[AMR08] Pascal Auscher, Alan McIntosh, and Emmanuel Russ, Hardy spaces of differential forms on Riemannian manifolds, J. Geom. Anal. 18 (2008), no. 1, 192-248.

[AvNP14] Pascal Auscher, Jan van Neerven, and Pierre Portal. Conical stochastic maximal $L^{p}$-regularity for $1 \leqslant p<\infty$. Math. Ann., 359(3-4):863-889, 2014.

[CMS83] R. R. Coifman, Y. Meyer, and E. M. Stein. Un nouvel éspace fonctionnel adapté à l'étude des opérateurs définis par des intégrales singulières. In Harmonic analysis (Cortona, 1982), volume 992 of Lecture Notes in Math., pages 1-15. Springer, Berlin, 1983.

[CMS85] R. R. Coifman, Y. Meyer, and E. M. Stein. Some new function spaces and their applications to harmonic analysis. J. Funct. Anal., 62(2):304-335, 1985.

[dS64] Luciano de Simon, Un'applicazione della teoria degli integrali singolari allo studio delle equazioni differenziali lineari astratte del primo ordine, Rend. Sem. Mat. Univ. Padova 34 (1964), 205-223.

[DY05] Xuan Thinh Duong and Lixin Yan. Duality of Hardy and BMO spaces associated with operators with heat kernel bounds. $J$. Amer. Math. Soc., 18(4):943-973 (electronic), 2005. 
"Hua-MaxRegHardy" — 2016/4/15 — 8:49 — page 12 — \#12

[HM09] Steve Hofmann and Svitlana Mayboroda, Hardy and BMO spaces associated to divergence form elliptic operators, Math. Ann. 344 (2009), no. 1, 37-116.

[HMM11] Steve Hofmann, Svitlana Mayboroda, and Alan McIntosh, Second order elliptic operators with complex bounded measurable coefficients in $L^{p}$, Sobolev and Hardy spaces, Ann. Sci. Éc. Norm. Supér. (4) 44 (2011), no. 5, 723-800.

[Hua15] Yi Huang, Operator theory on tent spaces, Thesis, Université Paris-Sud, Orsay, November 2015.

[Kat76] Tosio Kato. Perturbation theory for linear operators. SpringerVerlag, Berlin, second edition, 1976. Grundlehren der Mathematischen Wissenschaften, Band 132.

Yi HUANG

Laboratoire de Mathématiques d'Orsay

Univ. Paris-Sud, CNRS

Université Paris-Saclay

91405 Orsay, France

E-mail: Yi.Huang@math.u-psud.fr

Received April 15, 2016 